\title{
OVARIAN CARCINOMA WITH GALL BLADDER METASTASIS
}

\author{
Ambreen Zia, Ghulam Qadir Buledi and Syed Amir Dabir
}

\begin{abstract}
We report an unusual presentation of ovarian carcinoma, metastasizing to gall bladder. A 45 years old female, presented with diffuse abdominal pain and mass in the lower abdomen. On ultrasonography, she was found to have ovarian carcinoma with multiple tiny calculi in the gall bladder and thick irregular gall bladder walls. Laparotomy was done with oophrectomy and cholecystectomy. Histo-pathological examination revealed ovarian mucinous cyst adenocarcinoma along with ulcerated gall bladder walls showing metastatic deposits of adenocarcinoma.
\end{abstract}

\section{KEY WORDS}

Ovarian carcinoma. Ultrasonography. Metastasis. Adeno-carcinoma.

\section{INTRODUCTION}

Ovarian cancer (carcinoma) is the third most common cancer among women in Western countries, after breast and colo-rectal cancers. ${ }^{1}$ The population of Pakistan has also been reported to have one of the highest rates of ovarian cancer worldwide and it is the third common cancer in women after carcinoma of breast and carcinoma of cervix, and most frequent cause of deaths. ${ }^{2}$

A wide variety of tumors is seen in ovary than any other organ in the body, because the histological and embryological development of ovaries is complex. Patients with ovarian carcinoma usually present late with the symptoms either due to lack of specific symptoms or delayed referral by the primary care centers. ${ }^{3}$ The death rate can be reduced, if disease could be diagnosed at an early stage. In clinical practice, ovarian carcinomas are usually detected by bimanual pelvic examination, ultrasonography and tumor markers. For early diagnosis, the sensitivity and specificity are increased by combined modalities like ultrasonography and tumor markers. ${ }^{4}$ Many serological tumor markers are available to characterize the potential ovarian tumors and one of them is cancer associated antigen 125 (CA125). Through this paper, we report an unusual presentation of ovarian carcinoma, metastasizing to gall bladder.

\section{CASE REPORT}

A 45 years old female was referred to Radiology department from gynaecological out-patients department of Liaquat University Hospital Hyderabad, Sindh in July 2005. Patient had complaints of diffuse abdomi- nal pain, generalized weakness and mass in the lower abdomen since last 4-5 years. She also complained of irregular menses since 3-4 months. She was para 8+ 0 , all normal vaginal deliveries with history of tubal ligation 5 years back. There was no history of post coital bleeding. On general physical examination, she was anaemic while abdominal examination revealed a hard tender mass, dull on percussion, about 24 weeks size, in the lower abdomen. On per-vaginal examination, vulva and vagina were normal. No abnormality was also detected on cervical examination. Size of the uterus could not be assessed probably due to the mass. Fullness was felt in both fornices. There was no discharge or bleeding. Rest of the systemic examination was unremarkable. The significant laboratory findings in the complete blood picture included $\mathrm{Hb} 9.0 \mathrm{~g} / \mathrm{dl}$ and ESR $25 \mathrm{~mm}$ 1st hour (W. Green). Other routine investigations like blood sugar, serum urea, electrolytes and $x$-ray chest were within normal limits. She was referred for ultrasound pelvis, and both suprapubic and trans-vaginal ultrasound was carried out. Findings suggested a complex mass predominantly cystic with thick and irregular septations, internal echoes and solid components in the right adnexia measuring about $15 \mathrm{~cm}$ with irregular walls. Uterus was ante-verted, normal sized with central cavity echoes. No focal uterine mass was seen (Figure I). Doppler ultrasound was also carried out and the tumor vessels showed low resistance blood flow with Resistive Index Value less than 0.4 (Figure II). Findings were suggestive of a malignant ovarian mass. As a routine, ultrasound abdomen was carried out that showed $0.6 \mathrm{~cm}$ 
thick, hyper-echoic and irregular walls of the gall bladder. No focal mass was seen. Multiple tiny echogenic areas were also seen with the larger ones measuring about $0.5 \mathrm{~cm}$ in size. Common bile duct was found within normal limits (Figure III). Liver, spleen, both kidneys and urinary bladder were found within normal limits. No free fluid or enlarged lymph nodes were seen. Laparotomy was done with cholecystectomy and right-sided salpingo-oophrectomy.

Histo-pathological examination of the ovary on gross examination showed a large purplish brown ovarian tumor $(20 \times 15 \times 06 \mathrm{~cm})$ with broken surface at one place. Cut surface showed cystic area filled with thin light-brown gelatinous fluid and grey-white areas. A light brown fallopian tube was also attached $(07 \mathrm{~cm}$ long and $0.6 \mathrm{~cm}$ in diameter). Representative sections were taken in four blocks. Microscopically, ovary showed a mucinous cyst adeno-carcinoma and the capsule was infiltrated and at one place it appeared broken. The findings favoured "mucinous cyst adenocarcinoma of ovary". Histo-pathological findings of gall bladder on gross examination revealed a grey-brown partly opened gall bladder $(06 \times 2.5 \times 01 \mathrm{~cm}$.) Wall was up to $0.5 \mathrm{~cm}$ thick and the mucosa was ulcerated. Several light-brown stones $(0.5 \mathrm{~cm}$ in diameter) were also reported. Representative sections were taken in two blocks. Microscopically, gall bladder showed changes of chronic cholecystitis and in addition it was infiltrated by a mucinous adeno-carcinoma from ovary. It was concluded to be "gall bladder metastatic adenocarcinoma".

Patient developed the recurrence of tumor in ovary of left side though she received one cycle of chemotherapy.

\section{FIGURE I:}

\section{ULTRASOUND OF THE PELVIS SHOWING CYSTIC AND SOLID COMPONENTS AND THE UTERUS (ARROWS)}

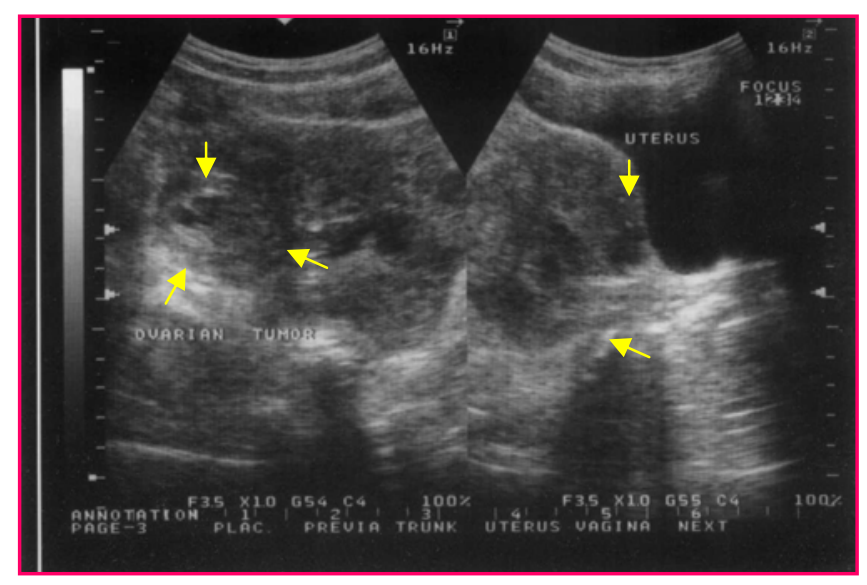

FIGURE II:
DOPPLER ULTRASOUND (COLOUR FLOW IMAGE) OF THE MASS WITH SOLID AREAS SHOWING VASCULARITY (ARROWS)

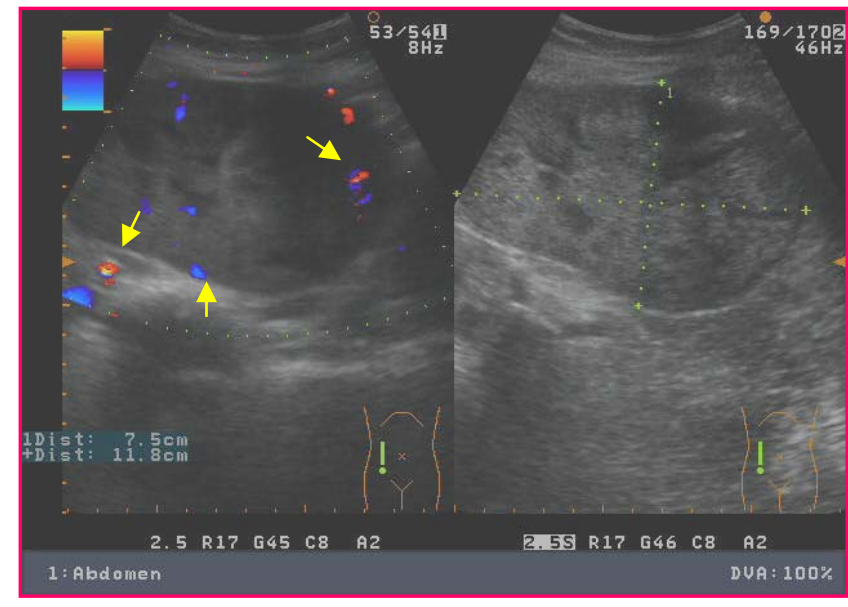

FIGURE III:

ULTRASOUND OF THE ABDOMEN SHOWING GALL BLADDER WITH THICK AND IRREGULAR WALL AND STONES AT NECK AND FUNDUS (ARROWS)

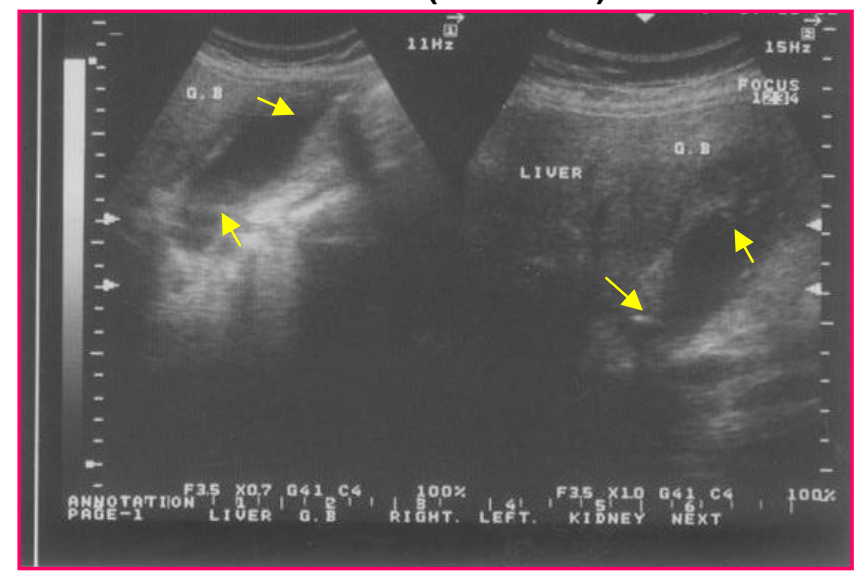

\section{DISCUSSION}

When ovarian cancer spreads beyond the ovaries, it generally does so either by seeding into the peritoneal cavity (including regional invasion) or by way of lymphatic dissemination. Retroperitoneal nodal spread may be present even in cases with little or no apparent intra-peritoneal involvement. Most of the lymphatic drainage of the ovary proceeds cephalad along the infundibulo-pelvic ligaments to the aortic nodal group. The intra-peritoneal spread of ovarian cancer is more apparent clinically than is the spread through lymphatic vessels. Haematogenous spread to parenchymal organs or bone occasionally occurs in advanced disease but is not significant in apparent early disease. $^{5}$ 
Common sites of ovarian metastases are liver, pleura, lung, central nervous system, skin, extra-abdominal lymph nodes, spleen, bone, and breast. ${ }^{6}$ Malignant mucinous tumors comprise $5-10 \%$ of malignant primary neoplasms. They occur most commonly between the fourth and the seventh decades of life. Although in $15-20 \%$ of cases, they are bilateral, most of them show extension beyond the ovaries at the time of Laparotomy. ${ }^{7}$

Mucinous cystadenocarcinoma of the ovary is commonly seen in Pakistani population and tend to occur at a younger age group. ${ }^{8}$

Ultrasonography is 80 to $90 \%$ accurate in gross morphology i.e. nature (solid versus cystic), size and location of pelvic masses but histological correlation is essential for diagnosis of ovarian masses. ${ }^{9,10}$ The importance of screening has increased because of the highest number of women suffering from ovarian malignancy present in advance stage when treatment usually fails and prognosis is poor. Therefore, it is important to diagnose the ovarian cancer in an early stage. However, gallbladder metastases are very rare and usually arise from malignant melanoma, renal cell carcinoma and cervical carcinoma. ${ }^{11}$

\section{REFERENCES}

1. Greenlee RT, Murray T, Bolden S, Wingo PA. Cancer statistics, 2000. Ca Cancer J Clin. 2000; 50: 7-33.

2. Murad A. Ovulation induction and ovarian tumors, the debate continues. J Pak Med Assoc. 1988; 48: 353-6.

3. Sultana A, Hasan S, Siddiqui QA. Ovarian tumors:
A five years retrospective study at Abbasi Shaheed Hospital, Karachi. Pakistan. J Surg. 2005; 21(1):37-40.

4. Schutter EM, Kenemans $P$, Sohn $C$, Kriston $P$, Crombash G, Western R, et al. Diagnostic value of pelvic examination, ultrasound, and serum CA 125 in postmenopausal women with ovarian mass: an international multi-centric study. Cancer. 1994; 74: 1398-1460.

5. Zaloudek C. The ovary. In: Gompel C, Silverberg SG, eds. Pathology in gynecology and obstetrics. Philadelphia, PA: Lippincott. 1994: Pp. 313-413.

6. Cormio G, Rossi C, Cazolla A, Resta L, Loverro G, Greco P, et al. Distant metastasis in ovarian carcinoma. Int J Gynecol Cancer. 2003; 13 (2):125-9.

7. Kurman RJ (Ed). Blaustein's Pathology of the Female Genital Tract (4th ed). New York: SpringerVerlag. 1994: Pp. 439-86.

8. Ahmad Z, Kayani N, Hasan S, Muzaffar S, Gill M. Histological pattern of ovarian neoplasma. J Pak Med Assoc. 2000; 50(12): 416-9.

9. Woodward J, Sohaey R, Mezzetti T.P. Endometriosis: radiological- pathologic correlation. Radiographic Jr. 2001;21:193-216.

10. Callen PW. Ultrasonography in obstetrics and gynecology, (4th ed). Philadelphia: WB Saunders. 2000: Pp. 781-813.

11. Doval DC, Bhatia K, Pavithran K, Sharma JB, Vaid AK, Hazarika D. Breast carcinoma with metastasis to the gallbladder: an unusual case report with a short review of literature. Hepatobiliary Pancreat Dis Int. 2006; 5(2):305-7.

\begin{tabular}{|l|}
\multicolumn{1}{c|}{ AUTHOR AFFILIATION: } \\
Dr. Ambreen Zia (Corresponding Author) \\
Senior Women Medical Officer \\
Department of Radiology and Imaging, \\
Liaquat University Hospital, Hyderabad - Pakistan. \\
E-mail: drambreenzia@hotmail.com \\
Dr. Ghulam Qadir Buledi \\
Assistant Professor, Department of Radiology and Imaging, \\
Liaquat University Hospital, Hyderabad - Pakistan. \\
Dr. Syed Amir Dabir \\
Senior Medical Officer \\
Sir C.J. Institute of Psychiatry, Hyderabad - Pakistan. \\
\hline
\end{tabular}

